\section{Decoding and Fusion in Distributed Detection Schemes with Unreliable Communications}

\author{
MARCO MARTALÒ \\ GIANLUIGI FERRARI \\ University of Parma
}

In the work presented here, we study how to combine decoding and fusion at the access point (AP) in sensor networks for distributed binary detection. We assume that all sensors make noisy observations of the same spatially constant binary phenomenon and communicate to the AP through noisy communication links. Simple distributed channel coding strategies are analyzed, either using repetition coding at each sensor (i.e., multiple observations) or distributed (network-wide) systematic block channel coding (possibly with local fusion in the presence of multiple observations). In the latter case, the use of a relay is proposed. In all cases, the system performance is analyzed separating or joining the decoding and fusion operations at the AP. As expected, the schemes with joint decoding and fusion show a significant performance improvement with respect to that of schemes with separate decoding and fusion.

Manuscript received April 9, 2009; revised April 20, 2010; released for publication November 8, 2010.

IEEE Log No. T-AES/48/1/943597.

Refereeing of this contribution was handled by C. Jauffret.

Authors' address: Wireless Ad-hoc and Sensor Networks (WASN) Laboratory, Dept. of Information Engineering, University of Parma, Viale Usberti 181/A, Parma, 43100, Italy, E-mail: (martalo@tlc.unipr.it).

$\overline{0018-9251 / 12 / \$ 26.00 ~(c) ~} 2012$ IEEE

\section{INTRODUCTION}

Sensor networks have been an active research field in the last years [1-3]. Communication-theoretic and information-theoretic approaches have been proposed for characterizing a sensor network with a spatially constant binary phenomenon under observation and direct communications between the sensors and the access point (AP) [4-8]. The performance of different fusion strategies in the presence of nonideal communication links has been also deeply investigated, especially in the realm of distributed detection $[9,10]$. One of the critical issues in designing sensor networks is their energy efficiency, especially in wireless scenarios, where sensors may be battery powered. Motivated by recent theoretical results in the area of network coding $[11,12]$, significant research activity has been devoted to the development of specific (distributed) channel coding strategies. Moreover, attention has also been dedicated, from an information-theoretic perspective, to distributed source coding, especially to the Gaussian central estimating officer (CEO) problem $[13,14]$.

In this paper we study simple distributed channel coding schemes applicable to sensor networks for decentralized detection of a spatially constant binary phenomenon. In particular, we apply well-known results of channel coding theory to sensor network scenarios, focusing on possible strategies for the combination of detection, decoding, and fusion operations ${ }^{1}$ at the AP. The fusion operation is restricted to be a simple majority-like fusion rule [8]. For simplicity, the term sensor refers to a wireless node which, besides sensing the phenomenon of interest, can also transmit. We use simple distributed channel codes, possibly relying on the presence of a proper intermediate relay node. We first consider scenarios where the observations at the sensors are ideal, i.e., error free, and we then extend our approach to scenarios where the sensors' observations are noisy, highlighting the relevant performance degradation caused by the observation noise in the presence of distributed channel coding. We also evaluate the performance degradation in the presence of noisy communication links from the sensors (and the relay) to the AP, and we comment on the impact of noisy links from the sensors to the relay (when used).

This paper is structured as follows. In Section II we provide the reader with preliminaries on decentralized detection (Section IIA) and on possible distributed channel coding strategies at the sensors

\footnotetext{
${ }^{1}$ In this paper, we use the term "detection" to indicate both the local processing on the received data at the input of the AP and the overall (cooperative) distributed processing of the network to detect the phenomenon status. The context will eliminate any ambiguity.
} 


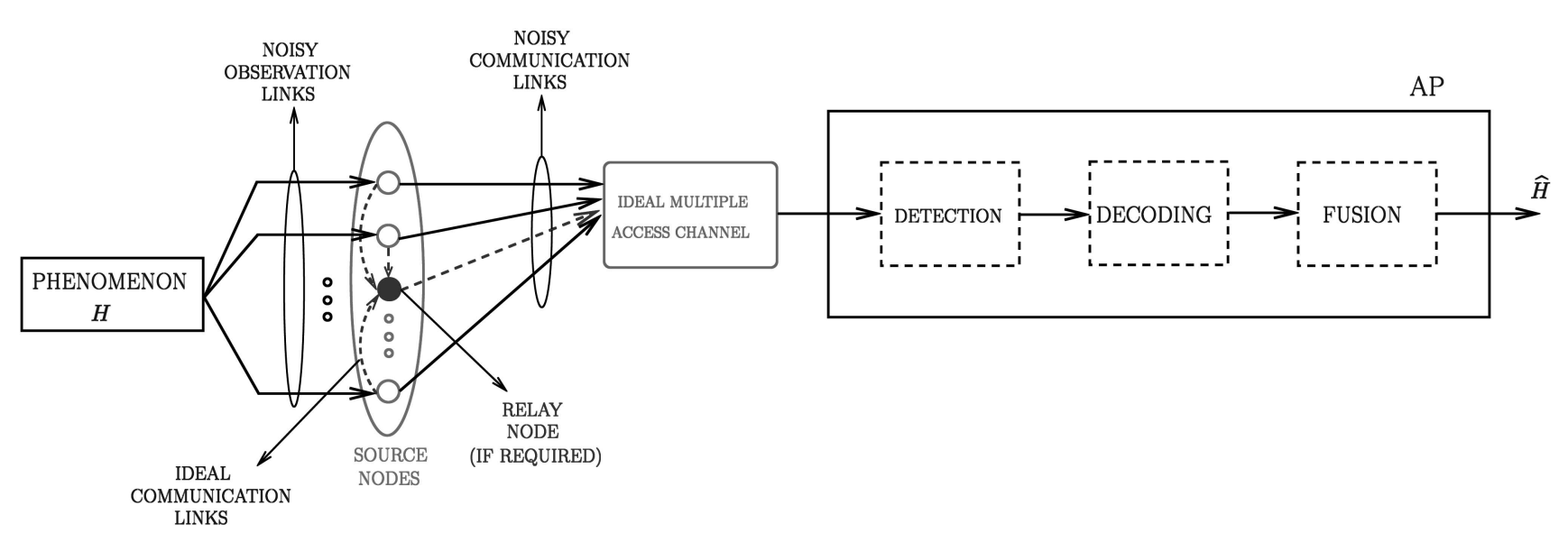

Fig. 1. Pictorial description of considered sensor network schemes. Solid lines are associated with mandatory elements (either blocks or connections), dashed lines associated with optional elements.

and detection/decoding/fusion strategies at the AP (Section IIB). In Section III, we study separate and joint decoding/fusion schemes in the presence of ideal observations at the sensors. In Section IV, our approach is extended to a scenario where the sensors' observations are noisy. In Section V, we discuss the impact of noisy communications between the sensors and the relay. Numerical results are presented in Section VI. Finally, Section VII concludes the paper.

\section{PRELIMINARIES}

\section{A. Decentralized Detection}

We consider a network scenario where $N$ sensors observe a spatially constant binary phenomenon whose status is defined as follows:

$$
H= \begin{cases}H_{0} & \text { with probability } p_{0} \\ H_{1} & \text { with probability } 1-p_{0} .\end{cases}
$$

In the remainder of this paper, we assume $p_{0}=1 / 2$, but our framework is applicable also to scenarios where $p_{0} \neq 1 / 2$. The assumption of a spatially constant binary phenomenon under observation has a practical validity when the goal of the sensor network is to detect if the observed phenomenon overcomes, on average, a critical threshold (e.g., detecting that the concentration of a dangerous gas is above a critical threshold) [15]. The observed signal at the $i$ th sensor can be expressed as

$$
y_{i}=c_{\mathrm{E}}+n_{i}, \quad i=1, \ldots, N
$$

where

$$
c_{\mathrm{E}} \triangleq \begin{cases}0 & \text { if } \quad H=H_{0} \\ s & \text { if } \quad H=H_{1}\end{cases}
$$

and $\left\{n_{i}\right\}$ are additive noise samples. Assuming that the noise samples $\left\{n_{i}\right\}$ are independent with the same Gaussian distribution $\mathcal{N}\left(0, \sigma^{2}\right)$, the common signal-to-noise ratio (SNR) at the sensors can be defined as follows: ${ }^{2}$

$$
\mathrm{SNR}_{\text {sensor }}=\frac{\left[\mathbb{E}\left\{c_{\mathrm{E}} \mid H_{1}\right\}-\mathbb{E}\left\{c_{\mathbb{E}} \mid H_{0}\right\}\right]^{2}}{\sigma^{2}}=\frac{s^{2}}{\sigma^{2}} .
$$

Each sensor makes a decision comparing its observation $y_{i}$ with a threshold value $\tau_{i}$ and computes a local decision $u_{i}=U\left(y_{i}-\tau_{i}\right)$, where $U(\cdot)$ is the unit step function. In order to optimize the system performance, the thresholds $\left\{\tau_{i}\right\}$ need to be optimized. Imposing that $\tau_{i}=\tau \forall i$, in all considered cases the optimized value of the common threshold $\tau$ is around $\sqrt{\mathrm{SNR}_{\text {sensor }}} / 2$, as already observed in $[8,17]$.

\section{B. Distributed Channel Coding and Detection/Decoding/Fusion Strategies}

In Fig. 1, a pictorial description of the considered sensor network model is shown. There are source nodes (the sensors), which observe (in a noisy manner) a spatially constant phenomenon and send their decisions to the AP, possibly using channel coding. The presence of a relay is also considered and a simple relaying strategy is proposed. The impact of multiple access interference is not investigated here: in other words, we assume orthogonal transmissions to the AP (e.g., perfect transmission scheduling between the sensors and, if present, the relay). The AP performs the following operations: detection of the observables, taking into account their statistical characterization; decoding of the embedded error correction code (when used); fusion of the decoded data to estimate the status of the phenomenon under observation. Note that some of the elements in Fig. 1 are present only in specific scenarios, for instance, the relay node and the decoding block in the AP appear only in coded scenarios.

In the following, we resort to classical communication theory in order to derive optimum

\footnotetext{
${ }^{2}$ The extension of the presented framework to scenarios with nonconstant observation SNR can be carried out following the approach proposed in [16].
} 
(in a maximum a posteriori probability (MAP) sense) decoding and fusion strategies at the AP.

1) Repetition Coded Sensor Network: A sensor network with multiple observations $(M$ consecutive and independent observations of the same phenomenon) can be interpreted as a system embedding a repetition code (with code rate $\left.R_{\mathrm{c}}=1 / M\right)$ at each sensor. In this case, redundant information is not sent by a relay, but from the sensors themselves through $M$ consecutive transmission acts per sensor.

2) Systematic Block Coded Sensor Network: In order to embed a systematic block channel code into a sensor network, we propose a simple relaying strategy. More precisely, we preliminary assume that each sensor makes a single observation, transmits its (uncoded) decision to the AP, and, owing to the broadcast nature of the wireless medium, also to the relay. Upon reception of the decisions from the sources, the relay, by using a systematic block code, generates parity bits and sends them to the AP. For example, an $(n, k)=(7,4)$ systematic Hamming code $[18$, p. 562] can be embedded into a sensor network with $N=k=4$ sensors and one relay, which generates $L=n-k=3$ bits according to the parity-check equations of the Hamming code. Assuming (as mentioned) that each sensor can reach both the AP and the relay in a single transmission act, the total number of transmission acts in the proposed sensor network is $N+L$. The equivalent code rate of this distributed coded scheme is $R_{\mathrm{c}}=$ $N /(N+L)=4 / 7$. Note, however, that the connections between the sensors and the relay have to be ideal (i.e., with no communication noise) in order for the proposed schemes to be applicable. This assumption is reasonable provided that, for example, the relay is relatively closer to the sensors than the AP is. In Section V, we comment on the impact of the noise in the communication links from the sensors to the relay.

In the presence of noisy observations, considering multiple observations and local fusion (at each sensor) might be beneficial in block coded scenarios. In fact, the "quality" of the transmitted information should improve, thus making channel coding more effective against communication noise. More details are given in Section IV. Note also that the use of multiple observations and local fusion in a scenario with ideal (noiseless) observations does not bring any benefit.

3) Communication Schemes: In a (repetition or block) coded scenario with binary phase shift keying (BPSK), the observable $r_{i}$ received at the output of an additive white Gaussian noise (AWGN) link between the $i$ th node and the AP can be written as

$$
r_{i}=\left(2 c_{i}-1\right) \sqrt{E_{\mathrm{c}}}+w_{i}, \quad i=1, \ldots, N+L
$$

where $c_{i} \in\{0,1\}$ is the symbol transmitted from either a sensor $\left(c_{i}\right.$ is an information bit, $\left.i=1, \ldots, N\right)$ or the relay (in this case, $c_{i}$ is a parity bit, $i=N+$ $1, \ldots, N+L),\left\{w_{i}\right\}$ are statistically independent AWGN samples with the same distribution $\mathcal{N}\left(0, N_{0} / 2\right), N_{0}$ being the single-sided noise power spectral density, and $E_{\mathrm{c}} \triangleq R_{\mathrm{c}} E_{\mathrm{b}}$ is the energy per coded bit, $E_{\mathrm{b}}$ being the energy per information bit. The link bit error rate (BER) at the output of the detector at the AP can be written as [19]

$$
p^{\mathrm{AWGN}}=Q\left(\sqrt{2 R_{\mathrm{c}} \gamma_{\mathrm{b}}}\right)
$$

where $Q(x) \triangleq \int_{x}^{\infty}(1 / \sqrt{2 \pi}) \exp \left(-y^{2} / 2\right) d y$ and $\gamma_{\mathrm{b}} \triangleq E_{\mathrm{b}} / N_{0}$ is the SNR at the AP.

In a coded scenario with BPSK and Rayleigh faded links, the observable at the output of the communication channel can be expressed as

$$
r_{i}=f_{i}\left(2 c_{i}-1\right) \sqrt{E_{\mathrm{c}}}+w_{i}, \quad i=1, \ldots, N+L
$$

where $f_{i}$ is a random variable with Rayleigh distribution-perfectly coherent demodulation is considered. Under the assumptions of independence between consecutive fading samples (e.g., through the use of channel interleaving) and that $\mathbb{E}\left[\left|f_{i}\right|^{2}\right]=1$, the link BER at the output of the detector at the AP is [19]

$$
p^{\text {Rayleigh }}=\frac{1}{2}\left[1-\sqrt{\frac{R_{\mathrm{c}} \gamma_{\mathrm{b}}}{1+R_{\mathrm{c}} \gamma_{\mathrm{b}}}}\right] .
$$

In general, one can denote as $p$ the link BER at the output of the detector, where $p$ has a specific expression (either (2) or (4)), depending on the communication scheme of interest, i.e., coding strategy and type of channel. For simplicity, we assume that $p$ is the same for all sensor-AP links.

In all communication schemes, the probability of decision error at the AP can be written, using the total probability theorem [20], as

$$
\begin{aligned}
P_{\mathrm{e}} & \triangleq P(\hat{H} \neq H) \\
& =p_{0} P\left(\hat{H}=H_{1} \mid H=H_{0}\right)+\left(1-p_{0}\right) P\left(\hat{H}=H_{0} \mid H=H_{1}\right) \\
& =\frac{1}{2}\left[P\left(\hat{H}=H_{1} \mid H=H_{0}\right)+P\left(\hat{H}=H_{0} \mid H=H_{1}\right)\right]
\end{aligned}
$$

where the conditional probabilities $P\left(\hat{H}=H_{i} \mid H=H_{j}\right)$ $(i, j=0,1, i \neq j)$ depend on the presence/absence of channel coding and on the detection/decoding/fusion strategy at the AP, as described in the following sections, distinguishing on the basis of the observations at the sensors.

\section{IDEAL OBSERVATIONS AT THE SENSORS}

In order to obtain performance benchmarks, we first consider scenarios where the spatially constant phenomenon $H$ is ideally detected by the sensors. In 
this case, we distinguish between AP structures where the decoding and fusion operations are either separate or joint.

\section{A. Separate Decoding and Fusion}

When the decoding and fusion operations are separate, assuming majority-like fusion the conditional probabilities at the right-hand side of (5) can be computed as follows:

$$
\begin{aligned}
& P\left(\hat{H}=H_{1} \mid H=H_{0}\right)=\sum_{i=k}^{N}\left(\begin{array}{c}
N \\
i
\end{array}\right)\left(p_{\mathrm{ch}}^{\text {ideal }}\right)^{i}\left(1-p_{\mathrm{ch}}^{\text {ideal }}\right)^{N-i} \\
& P\left(\hat{H}=H_{0} \mid H=H_{1}\right)=\sum_{i=0}^{k-1}\left(\begin{array}{c}
N \\
i
\end{array}\right)\left(1-p_{\mathrm{ch}}^{\text {ideal }}\right)^{i}\left(p_{\mathrm{ch}}^{\text {ideal }}\right)^{N-i}
\end{aligned}
$$

where the repeated trials formula has been used [20], $k$ (i.e., the majority decision threshold) is $\lfloor N / 2\rfloor+1$, and the probability $p_{\mathrm{ch}}^{\text {ideal }}$ depends on the noisy communication link model and the specific distributed channel coding strategy. Note that the upper index of the sum in (6) is $N$ (and not $N+L$ ) also in coded scenarios, since the information from the relay (i.e., the parity bits) is not used in the fusion process (only the systematic bits are used). The parity bits are used only in the detection/decoding process.

Since the local sensors' decisions are error free, $p_{\mathrm{ch}}^{\text {ideal }}$ and $1-p_{\mathrm{ch}}^{\text {ideal }}$ in (6) and (7) correspond to the probabilities of error and correct link decision at the detector output, respectively. In an "uncoded scenario" (i.e., $L=0$ ), it holds that $p_{\mathrm{ch}}^{\text {ideal }}=p$. In a scenario with multiple observations and repetition coding, the AP preliminary decides for the phenomenon status at each sensor through a majority fusion rule over the $M$ consecutive decisions sent by that sensor. In this case, $p_{\mathrm{ch}}^{\text {ideal }}$ can be expressed, similarly to (6), as

$$
p_{\mathrm{ch}}^{\text {ideal }}=\sum_{i=k_{\mathrm{NC}}}^{M}\left(\begin{array}{c}
M \\
i
\end{array}\right) p^{i}(1-p)^{M-i}
$$

where $k_{\mathrm{NC}} \triangleq\lfloor M / 2\rfloor+1$. In a block coded scenario and for sufficiently small values of $p$, the following approximation holds [18]:

$$
p_{\mathrm{ch}}^{\text {ideal }} \simeq\left(\begin{array}{c}
N+L-1 \\
t
\end{array}\right) p^{t+1}
$$

where $t=\left(d_{\min }-1\right) / 2$ is the number of errors which can be corrected by a code with minimum distance $d_{\min }[18,21]$. We point out that, provided that $1 / M=$ $N /(N+L)$, the comparison between repetition coded (with multiple observations) and block coded schemes is consistent from an energetic viewpoint.

\section{B. Joint Decoding and Fusion}

In a scenario with multiple $(M)$ independent observations at the sensors and repetition coding, joining the decoding and fusion operations consists in adopting a majority fusion rule over all the $N \times M$ bits sent from the sensors to the AP. In this case, the probability of decision error becomes

$$
\begin{aligned}
P_{\mathrm{e}}^{\text {rep. coding }}=\frac{1}{2}\left[\sum_{i=k_{\mathrm{M}}}^{N \times M}\left(\begin{array}{c}
N \times M \\
i
\end{array}\right) p^{i}(1-p)^{N \times M-i}\right. \\
\left.+\sum_{i=0}^{k_{\mathrm{M}}-1}\left(\begin{array}{c}
N \times M \\
i
\end{array}\right)(1-p)^{i} p^{N \times M-i}\right]
\end{aligned}
$$

where $k_{\mathrm{M}} \triangleq\lfloor N \times M / 2\rfloor+1$ is the majority decision threshold.

In a block coded scenario with single observations at the sensors, a receiver with joint decoding and fusion can be designed as follows. Since the considered sensor networks embed systematic codes, we denote as $\left[u_{1}^{(j)}, \ldots, u_{N}^{(j)}, b_{1}^{(j)}, \ldots, b_{L}^{(j)}\right]$ the entire sequence of bits transmitted by the sensors $\left(u_{i}^{(j)}\right.$ from sensor $i)$ and the relay $\left(\left\{b_{i}\right\}_{i=1}^{L}\right.$ from the relay) in correspondence to the phenomenon status $H_{j}$ $(j=0,1)$. Note that in the current case with a spatially constant binary phenomenon and ideal observations at the sensors, $\left(u_{1}, \ldots, u_{N}\right)$ is either $(0, \ldots, 0)$ or $(1, \ldots, 1)$. In other words, in the presence of ideal observations, only two codewords, denoted as $\mathbf{c}^{(0)}$ and $\mathbf{c}^{(1)}$, are allowed-this does not hold with noisy observations, as discussed in Section IV. In particular, $\mathbf{c}^{(0)}=(0, \ldots, 0)$. In all cases considered in this paper, it will also hold that $\mathbf{c}^{(1)}=(1, \ldots, 1)$.

Given that decoding and fusion are joint, two possible detection strategies at the AP can be devised: hard-output detection is followed by (hard-input) joint decoding/fusion; detection, decoding, and fusion are all joined together. In the former case, the MAP joint decoding/fusion strategy can be formalized as

$$
\hat{H}=\underset{j=0,1}{\arg \max } P\left(\mathbf{c}^{(j)} \mid \mathbf{c}_{\mathrm{rx}}\right)=\underset{j=0,1}{\arg \max } P\left(\mathbf{c}_{\mathrm{rx}} \mid \mathbf{c}^{(j)}\right) P\left(\mathbf{c}^{(j)}\right)
$$

where $\mathbf{c}_{\mathrm{rx}}$ is the codeword at the output of the detector at the AP. Since only two codewords $\mathbf{c}^{(0)}$ and $\mathbf{c}^{(1)}$ are used, the a priori probability of the sequence $\mathbf{c}^{(j)}$ is equal to the a priori probability of the phenomenon status $H_{j}$, i.e., $P\left(\mathbf{c}^{(j)}\right)=p_{j}=1 / 2$. Owing to the independence of the communication channels (conditionally on the transmitted bits), the MAP decoding/fusion strategy in (10) can be 
rewritten as

$$
\begin{aligned}
\hat{H} & =\underset{j=0,1}{\arg \max } p_{j} \prod_{i=1}^{N+L} P\left(c_{i, \mathrm{rx}} \mid c_{i}^{(j)}\right) \\
& =\underset{j=0,1}{\arg \max } \underbrace{\prod_{i=1}^{N+L}\left[(1-p)+(2 p-1)\left|c_{i, \mathrm{rx}}-c_{i}^{(j)}\right|\right]}_{Y^{(j)}\left(\mathbf{c}_{\mathrm{rx}}\right)}
\end{aligned}
$$

where the irrelevant term $p_{j}=1 / 2$ has been discarded and $p$ depends on the communication channel. After a few manipulations, $Y^{(j)}\left(\mathbf{c}_{\mathrm{rx}}\right)$ can be expressed as

$$
Y^{(j)}\left(\mathbf{c}_{\mathrm{rx}}\right)=p^{\vartheta\left(j, \mathbf{c}_{\mathrm{rx}}\right)}(1-p)^{N+L-\vartheta\left(j, \mathbf{c}_{\mathrm{rx}}\right)}, \quad j=0,1
$$

where $\vartheta\left(j, \mathbf{c}_{\mathrm{rx}}\right)$ denotes the number of bits in error at the AP when the phenomenon status is $H_{j}$ and $\mathbf{c}_{\mathrm{rx}}$ is the received sequence (at the output of the AP detector). In other words, $\vartheta\left(1, \mathbf{c}_{\mathrm{rx}}\right)$ is the number of $0 \mathrm{~s}$ and $\vartheta\left(0, \mathbf{c}_{\mathrm{rx}}\right)=N+L-\vartheta\left(1, \mathbf{c}_{\mathrm{rx}}\right)$ is the number of $1 \mathrm{~s}$ in $\mathbf{c}_{\mathrm{rx}}$. The MAP decoding/fusion strategy in (11) can be finally formulated as

$$
\left(\frac{1-p}{p}\right)^{2 \vartheta\left(1, \mathrm{c}_{\mathrm{r} x}\right)-N-L} \underset{H_{1}}{\stackrel{H_{0}}{\gtrless}} 1 .
$$

At this point, one can evaluate the probability of decision error in (5). In particular, the terms $\{P(\hat{H}=$ $\left.\left.H_{i} \mid H=H_{j}\right)\right\}(i, j=0,1, i \neq j)$ can be computed from the decision rule (12). After a few manipulations, one obtains detection/decoding/fusion strategy can be formulated as $^{3}$ [21]

$$
\begin{aligned}
\hat{H} & =\underset{j=0,1}{\arg \max } p\left(\mathbf{r} \mid \mathbf{c}^{(j)}, \mathbf{f}\right) P\left(\mathbf{c}^{(j)} \mid \mathbf{f}\right) \\
& =\underset{j=0,1}{\arg \max } p_{j} \prod_{i=1}^{N+L} p\left(r_{i} \mid c_{i}^{(j)}, f_{i}\right)
\end{aligned}
$$

where we have used the facts that the observables are conditionally independent given $\left\{c_{i}^{(j)}\right\}$ and the coded bit $c_{i}^{(j)}$ is independent of the fading sample $f_{i}$. Discarding $p_{j}=1 / 2$, from (13) one can derive the following binary decision rule:

$$
\prod_{i=1}^{N+L} \frac{p\left(r_{i} \mid c_{i}^{(0)}, f_{i}\right)}{p\left(r_{i} \mid c_{i}^{(1)}, f_{i}\right)} \underset{H_{1}}{\gtrless} 1
$$

where, according to (3), the probability density function (pdf) of the $i$ th observable $(i=1, \ldots, N+L)$ is

$$
p\left(r_{i} \mid c_{i}^{(j)}, f_{i}\right)=\frac{1}{\sqrt{\pi N_{0}}} \exp \left\{-\frac{\left(r_{i}-f_{i}\left(2 c_{i}^{(j)}-1\right) \sqrt{E_{\mathrm{c}}}\right)^{2}}{N_{0}}\right\}
$$

where $j=0,1$. By substituting (15) into (14), after a few manipulations the decision rule can be expressed as

$$
\sum_{i=1}^{N+L} r_{i} f_{i} c_{i}^{(1)} \underset{H_{0}}{\stackrel{H_{1}}{\gtrless}} 0 .
$$

On the basis of (16) and recalling that a linear combination of Gaussian random variables is

$$
P_{\mathrm{e}}=\frac{1}{2}\left[\sum_{k=k^{*}}^{N+L}\left(\begin{array}{c}
N+L \\
k
\end{array}\right) p^{k}(1-p)^{N+L-k}+\sum_{k=0}^{k^{*}-1}\left(\begin{array}{c}
N+L \\
k
\end{array}\right)(1-p)^{k} p^{N+L-k}\right]
$$

where we have used the fact that $\vartheta\left(j, \mathbf{c}_{\mathrm{rx}}\right)$ is a binomial random variable with parameters $N+L$ and $p, \mathbf{c}^{(1)}=\mathbf{1}$, and $k^{*}$ is defined as follows: still a Gaussian random variable [20], after a few manipulations the probability of decision error at the AP (5) becomes

$$
P_{\mathrm{e}}=\frac{1}{2}\left[Q\left(2 \frac{\sqrt{R_{\mathrm{c}} E_{\mathrm{b}}} \sum_{i=1}^{N+L} f_{i} c_{i}^{(1)}}{\sqrt{N_{0} \sum_{i=1}^{N+L} f_{i}^{2}\left(c_{i}^{(1)}\right)^{2}}}\right)+\Phi\left(-2 \frac{\sqrt{R_{\mathrm{c}} E_{\mathrm{b}} \sum_{i=1}^{N+L} f_{i}\left(2 c_{i}^{(1)}-1\right) c_{i}^{(1)}}}{\sqrt{N_{0} \sum_{i=1}^{N+L} f_{i}^{2}\left(c_{i}^{(1)}\right)^{2}}}\right)\right]
$$

$$
k^{*}=\min \{1, \ldots, N+L\} \quad \text { s.t. }\left(\frac{1-p}{p}\right)^{2 k^{*}-N-L}>1 .
$$

In the case with joint detection/decoding/fusion, we first consider a scenario with Rayleigh faded links, and we denote by $\mathbf{f}=\left[f_{1}, \ldots, f_{N+L}\right]$ the fading samples and by $\mathbf{r}=\left[r_{1}, \ldots, r_{N+L}\right]$ the observables at the output of the communication links. Under the assumption of perfect channel state information at the AP, the MAP where $\Phi(x) \triangleq 1-Q(x)$. Observe that (17) depends on the particular sequence of fading samples $\left\{f_{i}\right\}$.

An expression for the probability of decision error in the case with AWGN links can be directly obtained

\footnotetext{
${ }^{3}$ In (13) and in the remainder of this paper, the uppercase $P$ is used to denote the probability of an event, whereas the lowercase $p$ is used to denote the conditional probability density function (pdf) of a random variable.
} 
from (17) by imposing $f_{i}=1(i=1, \ldots, N+L)$. In particular, in the presence of a code with $\mathbf{c}^{(1)}=\mathbf{1}$ (recall that, in all cases, $\mathbf{c}^{(0)}=\mathbf{0}$ ) it can be shown that

$$
P_{\mathrm{e}}=Q\left(\sqrt{2(N+L) R_{\mathrm{c}} \gamma_{\mathrm{b}}}\right)=Q\left(\sqrt{2 N \gamma_{\mathrm{b}}}\right)
$$

\section{NOISY OBSERVATIONS AT THE SENSORS}

We now extend the derivation presented in Section III to encompass the presence of observation noise.

\section{A. Separate Decoding and Fusion}

In the case with separate decoding and fusion, only the expressions of the probabilities $p_{\mathrm{ch}}^{\text {ideal }}$ in (6) and (7) need to be modified. In particular, by using the total probability theorem [20], one can write

$$
\begin{aligned}
p_{\mathrm{ch}}^{\text {noisy }}= & P\left(c_{i, \mathrm{rx}}=1 \mid H_{\ell}\right) \quad \forall i=1, \ldots, N \\
= & P\left(c_{i, \mathrm{rx}}=1 \mid c_{i}^{(\ell)}=1, H_{\ell}\right) P\left(c_{i}^{(\ell)}=1 \mid H_{\ell}\right) \\
& +P\left(c_{i, \mathrm{rx}}=1 \mid c_{i}^{(\ell)}=0, H_{\ell}\right) P\left(c_{i}^{(\ell)}=0 \mid H_{\ell}\right) \\
= & p_{\mathrm{ch}}^{\text {ideal }} \Phi(\tau-s \cdot \ell)+\left(1-p_{\mathrm{ch}}^{\text {ideal }}\right) Q(\tau-s \cdot \ell)
\end{aligned}
$$

where the sensors' decisions $\left\{c_{i}^{(\ell)}\right\}$ are done as outlined in Section IIA and $p_{\mathrm{ch}}^{\text {ideal }}$ is the final BER, which depends on the presence/absence of distributed channel coding, as shown in Section III.

In a scenario with repetition coding and $M$ observations at each sensor, expression (8) for $p_{\mathrm{ch}}^{\text {ideal }}$ has to be similarly modified. In particular, one obtains

$$
p_{\mathrm{ch}}^{\text {noisy }}=\sum_{i=k_{\mathrm{NC}}}^{M}\left(\begin{array}{c}
M \\
i
\end{array}\right)[g(p, \ell)]^{i}[1-g(p, \ell)]^{M-i}
$$

where $g(p, \ell) \triangleq p \Phi(\tau-s \cdot \ell)+(1-p) Q(\tau-s \cdot \ell)$.

In the presence of multiple $(M)$ observations and block channel coding, the following strategy can be devised. Assuming that each sensor takes $M$ consecutive decisions (one per observation) and fuses them with a majority-like fusion rule, it is sufficient to modify the expression of $p_{\text {ch }}^{\text {noisy }}$ in (18). In particular, by using the total probability theorem, one obtains

$$
\begin{aligned}
p_{\mathrm{ch}}^{\text {noisy }} & =P\left(c_{i, \mathrm{rx}}=1 \mid H_{\ell}\right) \quad \forall i=1, \ldots, N \\
& =\left(1-p_{\mathrm{ch}}^{\text {ideal }}\right) \Psi(M, \tau, \ell)+p_{\mathrm{ch}}^{\text {ideal }}[1-\Psi(M, \tau, \ell)]
\end{aligned}
$$

where

$$
\Psi(M, \tau, \ell) \triangleq \sum_{i=k_{\mathrm{NC}}}^{M}\left(\begin{array}{c}
M \\
i
\end{array}\right)[Q(\tau-s \cdot \ell)]^{i}[\Phi(\tau-s \cdot \ell)]^{M-i} .
$$

At this point, the term (20) can be used, instead of (18), in (6) and (7). Note that the channel coding approach proposed in Section IIB2 can be interpreted as a multiple observations scheme, where $M-1$ (over $M$ ) observations are discarded.

\section{B. Joint Decoding and Fusion}

In the case with hard-output detection followed by joint decoding/fusion, expression (9) for a scenario with repetition coding and multiple observations at the sensors has to be modified, similarly to the derivation in Section IVA, as follows:

$$
\begin{aligned}
P_{\mathrm{e}, \text { noisy }}^{\text {mult. obs. }}= & \frac{1}{2} \sum_{i=k_{\mathrm{M}}}^{N \times M}\left(\begin{array}{c}
N \times M \\
i
\end{array}\right)[g(p, 1)]^{i}[1-g(p, 1)]^{N \times M-i} \\
& +\frac{1}{2} \sum_{i=0}^{k_{\mathrm{M}}-1}\left(\begin{array}{c}
N \times M \\
i
\end{array}\right)[1-g(p, 0)]^{i}[g(p, 0)]^{N \times M-i} .
\end{aligned}
$$

We now derive the MAP decoding/fusion strategies for block coded scenarios. In the case with hard-output detection followed by (hard-input) joint decoding/fusion, in order to take into account the statistical characterization of the observation noise expression (10) has to be modified as follows:

$$
\begin{aligned}
\hat{H} & =\underset{j=0,1}{\arg \max } P\left(H_{j} \mid \mathbf{c}_{\mathrm{rx}}\right)=\underset{j=0,1}{\arg \max } P\left(\mathbf{c}_{\mathrm{rx}} \mid H_{j}\right) P\left(H_{j}\right) \\
& =\underset{j=0,1}{\arg \max } \prod_{i=1}^{N+L} P\left(c_{i, \mathrm{rx}} \mid H_{j}\right)
\end{aligned}
$$

where the irrelevant term $P\left(H_{j}\right)=p_{j}=1 / 2$ has been discarded and the probability $P\left(c_{i, \mathrm{rx}} \mid H_{j}\right)$ can be written, after a few manipulations, as

$P\left(c_{i, \mathrm{rx}} \mid H_{j}\right)=\left\{\begin{array}{r}(1-p) \Phi(\tau-s \cdot j)+p Q(\tau-s \cdot j) \\ \text { if } c_{i, \mathrm{rx}}=0 \\ p \Phi(\tau-s \cdot j)+(1-p) Q(\tau-s \cdot j) \\ \text { if } c_{i, \mathrm{rx}}=1 .\end{array}\right.$

In a block coded scenario with joint detection/decoding/fusion, the MAP estimation strategy (13) has to be modified as follows:

$$
\begin{aligned}
\hat{H} & =\underset{j=0,1}{\arg \max } P\left(H_{j} \mid \mathbf{r}, \mathbf{f}\right)=\underset{j=0,1}{\arg \max } P\left(\mathbf{r} \mid H_{j}, \mathbf{f}\right) P\left(H_{j}\right) \\
& =\underset{j=0,1}{\arg \max } \prod_{i=1}^{N+L} P\left(r_{i} \mid H_{j}, f_{i}\right)
\end{aligned}
$$

which can be rewritten, after a few manipulations, as

where

$$
\frac{\prod_{i=1}^{N+L} \Upsilon\left(0, r_{i}, f_{i}\right)}{\prod_{i=1}^{N+L} \Upsilon\left(1, r_{i}, f_{i}\right)} \underset{H_{1}}{\stackrel{H_{0}}{\gtrless}} 1
$$

$$
\begin{aligned}
\Upsilon\left(m, r_{i}, f_{i}\right) \triangleq & \Phi(\tau-m \cdot s) \exp \left(-2 \frac{r_{i} f_{i} \sqrt{E_{\mathrm{c}}}}{N_{0}}\right) \\
& +[1-\Phi(\tau-m \cdot s)] \exp \left(2 \frac{r_{i} f_{i} \sqrt{E_{\mathrm{c}}}}{N_{0}}\right) .
\end{aligned}
$$


(a)

No communication
noise between
sensors and relay

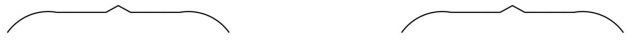

(b)

Communication noise between

sensors and relay

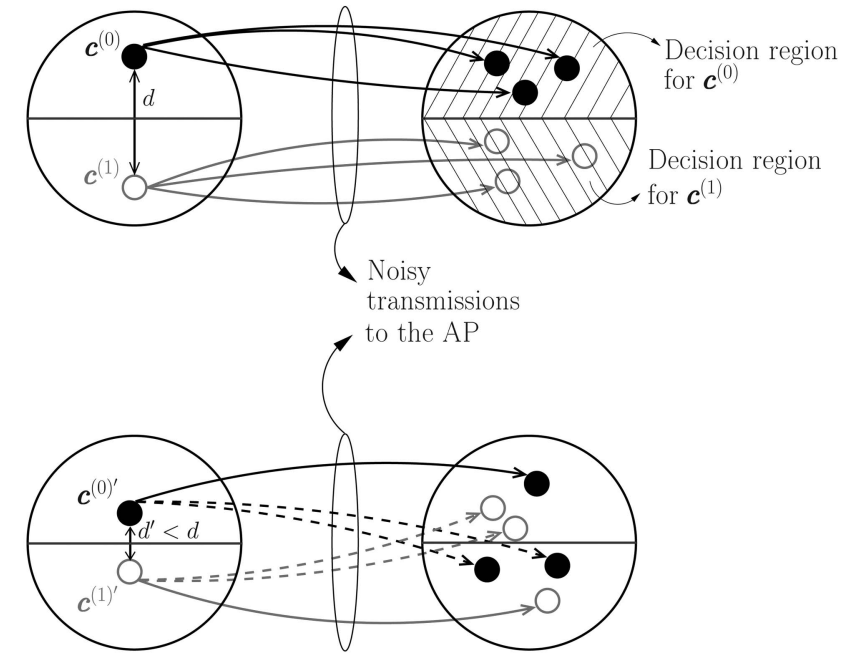

Fig. 2. Codebook perspective on considered distributed detection schemes. (a) Ideal communication links (no communication noise) between sensors and relay. (b) Noisy communication links. In each case, on the left the two possible codewords at sensors and relay are shown, whereas on the right possible received words at the AP are shown.

As considered in the presence of separate decoding and fusion, in the joint case with block channel coding one could consider multiple observations with local fusion as well. For the sake of conciseness, this is not considered.

\section{IMPACT OF NOISY COMMUNICATION LINKS TOWARDS THE RELAY}

The previous derivations in coded scenarios are based on the assumption of ideal communication links between the sensors and the relay. In this section, we briefly discuss the impact of noisy communication links between the sensors and the relay. No analytical derivation or numerical results are presented. The considerations which are carried out are simply meant to give some guidelines on the benefits brought by the distributed use of properly designed block error correction codes.

We first consider the case with ideal observations at the sensors. In Fig. 2 we give a pictorial description of how the communication noise influences data transmission to the relay. As previously seen, two possible codewords are selected at the sensors and relay, namely $\mathbf{c}^{(0)}$ and $\mathbf{c}^{(1)}$, which are shown in Fig. 2 as a filled circle and an empty circle, respectively. In the scenario with no communication noise between the sensors and the relay (Fig. 2(a)), we denote the Hamming distance between the two codewords as $d$. If $\mathbf{c}^{(0)}=\mathbf{0}$ and $\mathbf{c}^{(1)}=\mathbf{1}$, then $d=N+L$. The presence of noisy communication links from the sensors and the relay to the AP is such that the word $\mathbf{c}_{\mathrm{rx}}$ (one of the $2^{N+L}$ possible binary sequences of length $N+L$ ) received at the AP may be different from the codeword transmitted by the sensors and the relay. In particular, $\mathbf{c}_{\mathrm{rx}}$ may not even be a codeword. Carrying out decoding and fusion at the AP corresponds to associating the received word to one of the information sequences $\{\mathbf{0 , 1}\}$. It is intuitive that the larger $d$ is, the more robust the system is against noisy communication links between the sensors/relay and the AP.

In the presence of communication noise between the sensors and the relay (Fig. 2(b)), the latter may receive a sequence of bits which differs from that sent by the sensors. Therefore, the parity bits generated by the relay may lead to the association of $H_{0}$ and $H_{1}$ to two codewords $\mathbf{c}^{(0) \prime}$ and $\mathbf{c}^{(1) \prime}$ which are at a distance $d^{\prime}<d$. As a consequence of this decreased distance, the system performance will be worse than in the previous scenario, since the probability of associating (through decoding and fusion) the received word to the wrong phenomenon status will increase. This can be understood from the codebook scenario at the AP, where the received word at the AP might belong to the portion of the signal space which is associated (by decoding and fusion) to the wrong phenomenon status.

The presence of noisy observations may lead to the association of the phenomenon statuses $H_{0}$ and $H_{1}$ to two codewords $\mathbf{c}^{(0) \prime \prime}$ and $\mathbf{c}^{(1) \prime \prime}$ at a distance smaller than $d$. In particular, in the presence of both 1) observation noise and 2) communication noise from the sensors to the relay, when the intensities of these two noises are sufficiently small, their negative effects tend to add, so that the distance $d^{\prime \prime}$ between $\mathbf{c}^{(0) \prime \prime}$ and $\mathbf{c}^{(1) \prime \prime}$ might be even smaller than $d^{\prime}$.

Obviously, an open problem is to quantify precisely the decrease of the error correction capability $t$ of a code in the presence of noisy communication links between the sensors and the 


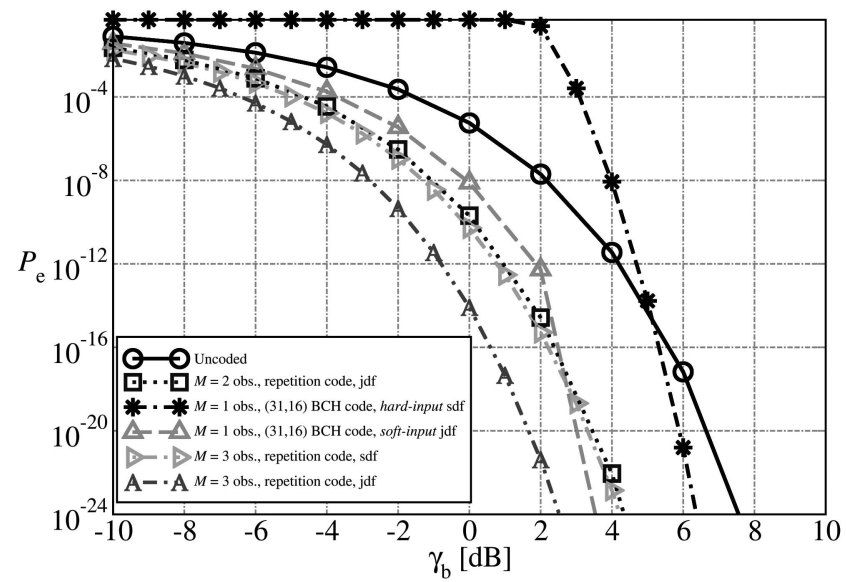

(a)

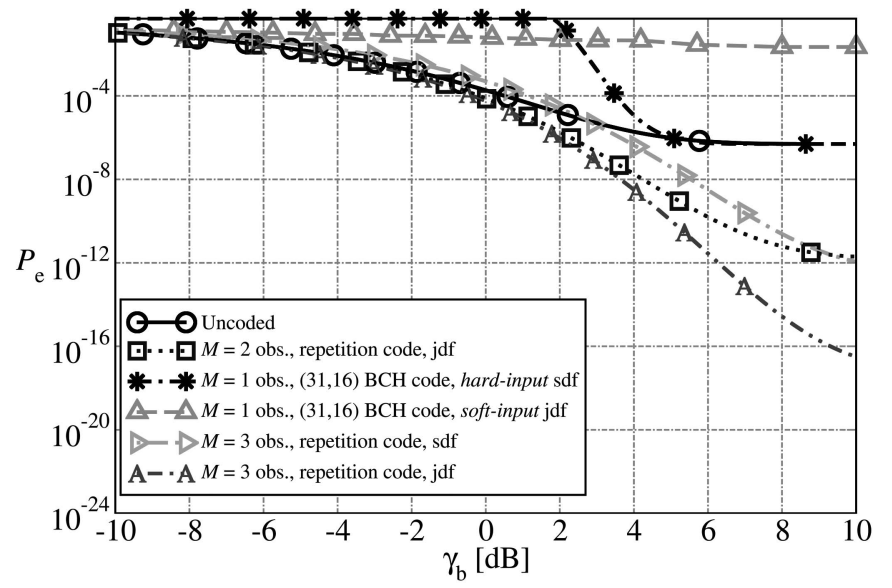

(b)

Fig. 4. Probability of decision error, as function of SNR at AP, in scenario with $N=16$ sensors and AWGN communication links. Two values for sensor SNR are considered: (a) $20 \mathrm{~dB}$ (error-free observations) and (b) $10 \mathrm{~dB}$ (noisy observations). Various coding strategies are considered.

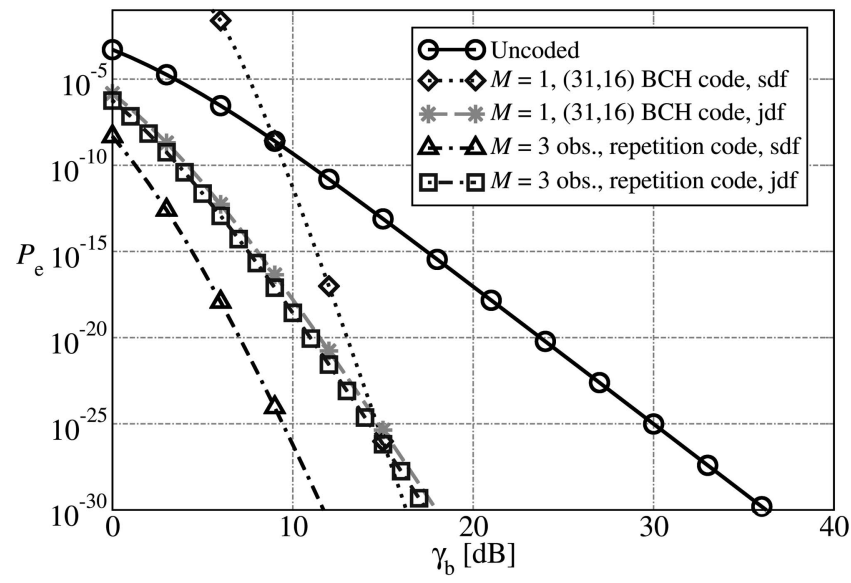

Fig. 3. Probability of decision error, as function of SNR at AP, in scenario with $N=16$ sensors, Rayleigh faded communication links, and error-free observations at sensors. Various sensor network architectures are considered.

relay. In fact, the parameter $t$ depends on the particular structure (codebook) of the considered error correction code. An interesting research direction, which goes beyond the scope of this paper, is the design of robust (fault tolerant) error correcting codes for the proposed distributed detection schemes.

\section{NUMERICAL RESULTS}

We now present numerical results associated with our analytical framework. In particular, as component channel codes, we consider some Bose, Chaudhuri, and Hocquenghem $(\mathrm{BCH})$ codes with different values of $t$ [21, p. 438]. ${ }^{4}$ In particular, the following $\mathrm{BCH}$ codes are considered: 1) $(31,16)$ with $t=3,2)(63,30)$ with $t=6$, and 3) $(127,64)$ with $t=10$.

\footnotetext{
${ }^{4}$ We remark that the $\mathrm{BCH}$ code is one of the block channel codes that it is possible to consider. However, the same results would be asymptotically obtained with any code with the same value of $t$.
}

In Fig. 3, the probability of decision error is shown, as a function of the SNR $\gamma_{b}$, in a scenario with $N=16$ sensors, error-free observations at the sensors, and Rayleigh faded communication links (according to the model in (3)). Five sensor network schemes are considered: 1 ) uncoded with single observations, 2) $(31,16) \mathrm{BCH}$ coded $[21$, p. 438] (the corresponding $\mathrm{BCH}$ code has $t=3$ ) with single observations at the sensors hard-input separate decoding/fusion, 3) $(31,16) \mathrm{BCH}$ coded with single observations at the sensors and hard-input joint decoding/fusion, 4) with $M=3$ observations, repetition coding, and separate decoding/fusion, and 5) with $M=3$ observations, repetition coding, and joint decoding/fusion. One can observe that the probability of decision error in case 2 shows a "waterfall" behavior, which is due to the concatenation of the decoding and fusion operations. However, the improvement brought by the presence of block channel coding, with respect to schemes with repetition, becomes apparent at very low probabilities of decision error, which may not be of practical interest.

In Fig. 4, the probability of decision error is shown, as a function of the SNR at the AP, in a scenario with $N=16$ sensors and AWGN communication links. Two values for the sensor SNR are considered: (a) $20 \mathrm{~dB}$ (basically error-free observations) and (b) $10 \mathrm{~dB}$ (noisy observations). Six coding strategies are considered: 1) uncoded, 2) $(31,16) \mathrm{BCH}$ coded with single observations at the sensors and hard-input separate decoding/fusion, 3) $(31,16) \mathrm{BCH}$ coded with single observations at the sensors and soft-input joint decoding/fusion, 4) with $M=2$ observations, repetition coding, and joint decoding/fusion, 5) with $M=3$ observations, repetition coding, and separate decoding/fusion, and 6) with $M=3$ observations, repetition coding, and joint decoding/fusion. In a scenario with ideal 
observations (case (a)), one can observe that the $\mathrm{BCH}$ coded network with soft-input joint decoding/fusion at the AP has a performance significantly better than that associated with the $\mathrm{BCH}$ coded schemes with hard-input separate decoding/fusion. This is to be expected, since in a scenario with soft-input decoding no information is lost upon reception of the observables from the communication links. Note, however, that the proposed block coded scheme outperforms a scheme with repetition coding only at very low values of the probability of decision error.

As can be observed comparing the results in Fig. 4(a) with those in Fig. 4(b), when the observation quality reduces the proposed detection/decoding/fusion strategies they become less effective, since the quality of the sensors' observations heavily affects the system performance, and this is more pronounced in the presence of joint decoding/fusion. One can also observe that the probability of decision error curve reaches a floor, due to the observation noise (which is independent of the communication noise). As before, the schemes with multiple observations at the sensors outperform those with block channel coding.

We now investigate the impact of multiple observations on the performance of block coded scenarios. In Fig. 5, the probability of decision error is shown, as a function of the SNR at the AP, in a scenario with $N=16$, AWGN communication links, and noisy phenomenon observations (with observation SNR set to $10 \mathrm{~dB}$ ). In all cases, separate decoding and fusion are considered. As can be seen from the obstained results, in the $\mathrm{BCH}$ coded case the impact of the use of multiple observations becomes noticeable for $\gamma_{\mathrm{b}} \gtrsim 2 \mathrm{~dB}$. In this SNR region, it can be observed that the performance with a given odd value of $M$ (either 1 or 3 ) degrades, relatively, when $M$ is increased to the consecutive even value. This is because in the presence of noisy observations, increasing the number of decisions to be fused from an odd number to the next even one does not improve the "intrinsic" quality of the majority-like fusion but, instead, degrades it-this degradation disappears if the observation noise reduces (e.g., the observation SNR is increased to $20 \mathrm{~dB}$ ). One should also note that, when the number of observations is sufficiently large (e.g., $M \geq 3$ ), the use of them becomes effective for the $\mathrm{BCH}$ coded scheme, which can outperform the corresponding (for the same value of $M$ ) repetition coded scheme. In particular, this effect is evident also for values of interest for the probability of decision error (e.g., $P_{\mathrm{e}} \simeq 10^{-6}$ ). However, for large values of $\gamma_{\mathrm{b}}$ the probability of decision error of block and repetition coded schemes reaches, for a given value of $M$, the same floor.

Finally, we investigate the performance of the proposed distributed detection/decoding schemes in large scale sensor networks, when $N$ is increased to 30 and 64 , by considering proper $\mathrm{BCH}$ codes. In

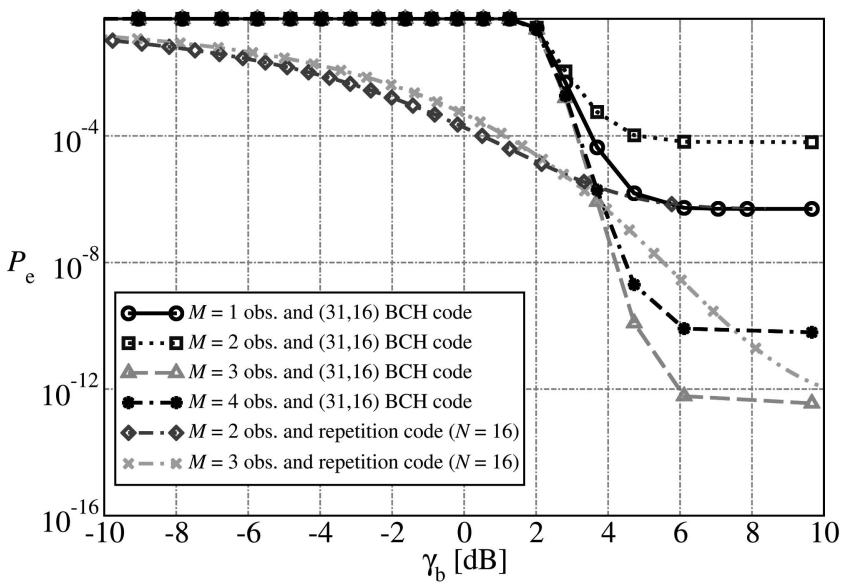

Fig. 5. Probability of decision error, as function of SNR at AP, in scenario with $N=16$, AWGN communication links, and noisy phenomenon observations (with observation SNR set to $10 \mathrm{~dB}$ ). Various coding strategies, with various numner of observations, are considered. In all cases, decoding and fusion are separate.

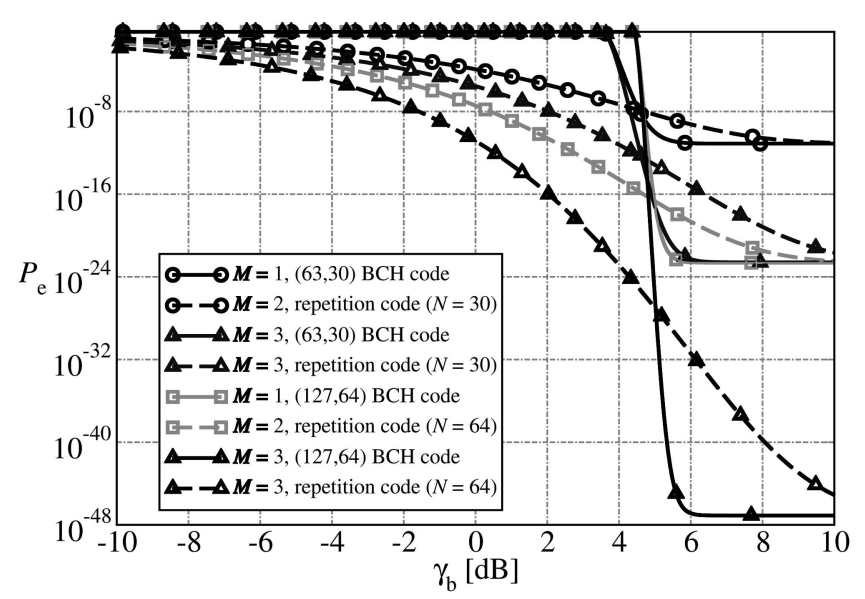

Fig. 6. Probability of decision error, as function of the SNR at AP, in scenario with AWGN communication links and noisy phenomenon observations (with observation SNR set to $10 \mathrm{~dB}$ ).

Various coding strategies with separate channel decoding and fusion are considered. Two values for number of sensors $N$ are considered, namely, $N=30$ and $N=64$.

Fig. 6, the probability of decision error is shown, as a function of the SNR at the AP, in a scenario with AWGN communication links and noisy phenomenon observations (with observation SNR set to $10 \mathrm{~dB}$ ). For each value of $N$, the performance of the system with multiple observations and $\mathrm{BCH}$ coding is compared with that associated with the same number of multiple observations and repetition coding. One can observe that for $\gamma_{\mathrm{b}} \gtrsim 4 \mathrm{~dB}$ the scheme with multiple observations and $\mathrm{BCH}$ coding becomes the winning strategy. This confirms the results shown in Fig. 5 also for large scale sensor networks. However, with respect to the scenario with a smaller value of $N$ (i.e., $N=16$ ) shown in Fig. 5, one can observe that this effect appears for lower values of the probability of decision error, which may not be of interest. Therefore, for large scale sensor networks repetition 
coding seems the winning strategy. We remark that in [22] large sensor networks are analyzed by resorting to low-density parity-check (LDPC) codes.

\section{CONCLUDING REMARKS}

In this paper, we have studied how to combine detection, decoding, and fusion at the AP in sensor networks for decentralized detection of a spatially constant binary phenomenon. To this end, we have embedded simple distributed channel codes (either block or repetition) into sensor network architectures. The performance of the proposed schemes has been analyzed in scenarios with noisy observations and communications. We have also considered the use of multiple observations at the sensors: without local fusion this leads to the use of repetition coding; in the presence of local fusion, block channel coding can then be considered. Repetition coding guarantees the best performance, with respect to simple systematic block coding strategies, when ideal observations at the sensors are considered. In fact, the best performance for practical values of the probability of decision error can be obtained. When the observation noise increases, the use of multiple observations with block channel coding becomes the winning strategy for intermediate SNR values. However, the block and repetition coded schemes approach the same limiting (for high channel SNR) probability of decision error. In large scale sensor networks, repetition coding is attractive for practical values of the probability of decision error.

\section{REFERENCES}

[1] Akyildiz, I., et al.

A survey on sensor networks.

IEEE Communications Magazine, 40, 8 (Aug. 2002), 102-114.

[2] Chong, C. Y. and Kumar, S. P.

Sensor networks: Evolution, opportunities, and challenges.

Proceedings of the IEEE, 91, 8 (Aug. 2003), 1247-1256.

[3] Simic, S. N. and Sastry, S.

Distributed environmental monitoring using random sensor networks.

In Proceedings of the 2nd International Workshop on Information Processing in Sensor Networks (IPSN), Palo Alto, CA, Apr. 2003, 582-592.

[4] Tenney, R. R. and Sandell, N. R. Detection with distributed sensors.

IEEE Transactions on Aerospace and Electronic Systems, AES-17, 4 (July 1981), 501-510.

[5] Viswanathan, R. and Varshney, P. K.

Distributed detection with multiple sensors-Part I: Fundamentals.

Proceedings of the IEEE, 85, 1 (Jan. 1997), 54-63.

[6] Hoballah, I. Y. and Varshney, P. K.

An information theoretic approach to the distributed detection problem.

IEEE Transactions on Information Theory, 35, 5 (Sept. 1989), 988-994.
[7] Luo, Z-Q.

An isotropic universal decentralized estimation scheme for a bandwidth constrained ad hoc sensor network. IEEE Journal on Selected Areas in Communications, 23, 4 (Apr. 2005), 735-744.

[8] Ferrari, G. and Pagliari, R.

Decentralized binary detection with noisy communication links.

IEEE Transactions on Aerospace and Electronic Systems, 42, 4 (Oct. 2006), 1554-1563.

[9] Chen, B. and Willett, P.

On the optimality of the likelihood-ratio test for local sensor decision rules in the presence of non-ideal channels.

IEEE Transactions on Information Theory, 51, 2 (Feb. 2005), 693-700.

[10] Mergen, G., Naware, V., and Tong, L.

Asymptotic detection performance of type-based multiple access over multiaccess fading channels.

IEEE Transactions on Signal Processing, 55, 3 (Mar. 2007), 1081-1092.

[11] Fragouli, C. and Soljanin, E.

Network Coding Fundamentals.

Hanover, MA: Now Publisher Foundations and Trends in Networking, 2007.

[12] Fragouli, C. and Soljanin, E.

Network Coding Applications.

Hanover, MA: Now Publisher Foundations and Trends in Networking, 2007.

[13] Berger, T. Zhang, Z., and Viswanathan, H.

The CEO problem.

IEEE Transactions on Information Theory, 42, 3 (May 1996), 887-902.

[14] Chen, J. and Berger, T.

Robust distributed source coding.

IEEE Transactions on Information Theory, 54, 8 (Aug. 2008), 3385-3398.

[15] Duffin, K., et al.

Chemical sensor networks for gas detection and environmental monitoring.

Current Analytical Chemistry, 4, 4 (Oct. 2008), 391-402.

[16] Ferrari, G., Pagliari, R., and Martalò, M.

Decentralized binary detection with non-constant SNR profile at the sensors.

International Journal on Sensor Networks (Special Issue on Energy-Efficient Algorithm and Protocol Design in Sensor Networks), 4, 1 (2008), 23-36.

[17] Shi, W., Sun, T. W., and Wesel, R. D.

Quasi-convexity and optimal binary fusion for distributed detection with identical sensors in generalized Gaussian noise.

IEEE Transactions on Information Theory, 47, 1 (Jan. 2001), 446-450.

[18] Carlson, A. B., Crilly, P. B., and Rutledge, J. C. Communication Systems, An Introduction to Signals and Noise in Electrical Communication (4th ed.). Columbus, OH: McGraw-Hill, 2002.

[19] Molisch, A. F.

Wireless Communications.

Hoboken, NJ: Wiley, 2005.

[20] Papoulis, A.

Probability, Random Variables and Stochastic Processes. Columbus, OH: McGraw-Hill, 1991.

[21] Proakis, J. G.

Digital Communications (4th ed.).

Columbus, OH: McGraw-Hill, 2001.

[22] Martalò, M. and Ferrari, G.

Decoding and fusion in sensor networks with noisy observations and communications.

In Proceedings of the 2008 IEEE International Symposium on Spread Spectrum Techniques and Applications (2008 ISSSTA), Bologna, Italy, Aug. 2008, 7-11. 


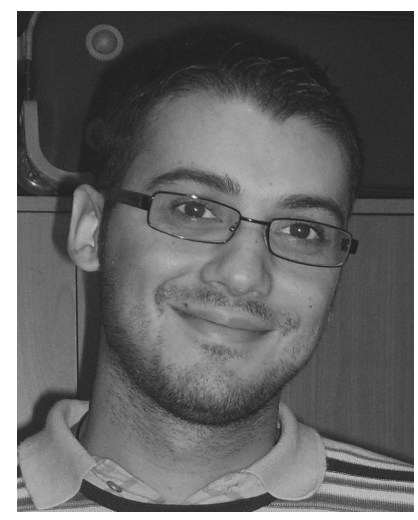

Marco Martalò was born in Galatina (LE), Italy, in June 1981. He received the "Laurea" degree (3-year program) and the "Laurea Specialistica" $(3+2$ year program) degree (summa cum laude) in telecommunications engineering in 2003 and 2005, respectively, from the University of Parma, Italy. In 2009 he received the Ph.D. degree in information technologies from the University of Parma, Italy.

Since January 2009, he is a Post-Doc researcher at the Information Engineering Department of the University of Parma, Italy. From October 2007 to March 2008, he was a "Visiting Scholar" at the School of Computer and Communication Sciences of the Ecole Polytechnique Federale De Lausanne (EPFL), Lausanne, Switzerland, collaborating with the Laboratory of Algorithmic Research in Network Information, directed by Professor Christina Fragouli. He is a member of the Information Engineering Department of the University of Parma, Italy, of the Wireless Ad-hoc and Sensor Networks (WASN) Laboratory.

He is coauthor of the book Sensor Networks with IEEE 802.15.4 Systems: Distributed Processing, MAC, and Connectivity (Springer, 2011). Dr. Martalò was a corecipient of a "best student paper award" (with his tutor Dr. Gianluigi Ferrari) at the 2006 International Workshop on Wireless Ad hoc Networks (IWWAN'06). $\mathrm{He}$ has been a TPC member of the IEEE Global Communications Conference (GLOBECOM 2011), the International Workshop on Performance Methodologies and Tools for Wireless Sensor Networks (WSNPERF 2009) and the International Conference on Advances in Satellite and Space Communications (SPACOMM 2009-2010). He also serves as reviewer for many international journals and conferences.

Gianluigi Ferrari was born in Parma, Italy, in 1974. He received his "Laurea" and Ph.D. degrees from the University of Parma, Italy, in 1998 and 2002, respectively.

Since 2002, he has been with the University Parma, where he currently is an Associate Professor of Telecommunications. He was a visiting researcher at USC (Los Angeles, CA, 2000-2001), CMU (Pittsburgh, PA, 2002-2004), KMITL (Bangkok, Thailand, 2007), and ULB (Bruxelles, Belgium, 2010). Since 2006, he has been the Coordinator of the Wireless Ad-hoc and Sensor Networks (WASN) Lab in the Department of Information Engineering of the University of Parma.

As of today Dr. Ferrari has published more than 140 papers in leading international journals and conferences. He is coauthor of a few books, including Detection Algorithms for Wireless Communications, with Applications to Wired and Storage Systems (Wiley, 2004), Ad Hoc Wireless Networks: A Communication-Theoretic Perspective (Wiley, 2006), LDPC Coded Modulations (Springer, 2009), and Sensor Networks with IEEE 802.15.4 Systems: Distributed Processing, MAC, and Connectivity (Springer, 2011). He edited the book, Sensor Networks: Where Theory Meets Practice (Springer, 2010). His research interests include digital communication systems analysis and design, wireless ad hoc and sensor networking, adaptive digital signal processing, and information theory.

Dr. Ferrari is a corecipient of a best student paper award at the 2006 International Workshop on Wireless Ad hoc Networks (IWWAN'06) and a best paper award at EMERGING'10. He acts as a frequent reviewer for many international journals and conferences. He acts also as a technical program member for many international conferences. He currently serves on the Editorial Boards of The Open Electrical and Electronic Engineering (TOEEJ) Journal (Bentham Publishers), the International Journal of RF Technologies: Research and Applications (Taylor \& Francis), and the International Journal of Future Generation Communication and Networking (SERSC: Science \& Engineering Research Support Center). He was a Guest Editor of the 2009 EURASIP JWCN Special Issue on "Dynamic Spectrum Access: From the Concept to the Implementation." 